\title{
Intra operative temporomandibular joint dislocation during use of proseal LMA
}

\author{
Zameer Farooq ${ }^{1, *}$, Neha Gupta ${ }^{2}$, Farah Nabi $^{3}$ \\ ${ }^{1}$ Senior Registrar, Dept. of Critical Care Medicine, Indraprasth Apollo Hospital, New Delhi, ${ }^{2}$ Senior Resident, Dept. of \\ Anaesthesia, Lok Nayak Hospital New Delhi, ${ }^{3}$ Lecturer, Dept. of Gynaecology and Obstetrics, Government Medical College, \\ Srinagar, Jammu and Kashmir, India
}

*Corresponding Author:

Email: zmrshah@gmail.com

Received: $9^{\text {th }}$ April, 2018

Accepted: $25^{\text {th }}$, April, 2018

\begin{abstract}
General anaesthesia is associated with several common complications, TMJ dislocation is one of the rare complications encountered during airway management. Usually occurs during endotracheal intubation but other factors such as force full laryngoscopy, manual attempt to complete intubation or duration for which TMJ was stressed may also be responsible. The incidence of TMJ dysfunction is reported to be $5 \%$ following endotracheal intubation, ${ }^{1}$ but less cases have been reported during LMA insertion. We report a case of TMJ dislocation in a 25 year old female patient during general anaesthesia after use of proseal LMA.
\end{abstract}

Keywords: Proseal LMA, TMJ, Isoflurane, Propofol, General anaesthesia.

\section{Introduction}

TMJ dislocation is a rare complication that can occur during endotracheal intubation, jaw thrust for LMA insertion, laryngoscopy and jaw manipulation during nasogastric tube insertion. TMJ instability is common among females of middle age group and 25 to 50 percent prevalence in general population. ${ }^{2,3}$ TMJ dislocation that occurs during surgeries under general anaesthesia is often unnoticed until patients become aware of pain and swelling. Unrecognized TMJ dislocation can lead to persistant pain and restricted joint mobility. ${ }^{4}$ Endotracheal intubation under anaesthesia has long back been considered as a risk factor for excecerbation or development of TMJ dysfunction. During intubation rotation and translation of the TMJ is attempted to allow for adequate mouth opening. This maneuver allows for adequate visualization of vocal cords and glottic structures, and consequently smooth endotracheal intubation. During this procedure, damage may occur to the TMJ apparatus due to excessive forces being applied either with the laryngoscope, or manually in an attempt to complete the intubation. Jaw thrust is used as an alternative technique when LMA insertion fails by conventional method. Forward jaw thurst during LMA insertion put strain on TMJ and can lead to dislocation.

\section{Case Report}

A 25 year old, $55 \mathrm{~kg}$, female patient, G3P2, diagnosed as 6 weeks pregnancy was posted for MTP. Patient had underwent uneventful general anaesthesia for elective laproscopic cholecystectomy 4 years back. Patient had no significant medical history. Investigations were with in normal limits. Airway assessment revealed mouth opening of $6 \mathrm{~cm}$, thyromental distance of $8 \mathrm{~cm}$, free neck movements,
MP grade 2 and no history of TMJ dislocation or any injury.

Patient was preoxygenated with $100 \%$ oxygen for 3 minutes and injection fentanyl 100 microgram intravenous was given. Injection propofol $100 \mathrm{mg}$ was given iv slowly. After achieving adequate muscle relaxation, proseal LMA of size 3 was inserted after giving jaw thrust. Bilateral air entry was checked and ryles tube of 12 french was inserted through gastric port of LMA. Inhalational anaesthesia was started with isoflurane at $1 \%$ and nitrous oxide and oxygen at $66 \%$ and $33 \%$ respectively. Patient was initially ventilated manually with bains circuit. After 5 minutes patient started breathing spontaneously. Intra operative period was uneventfull. At the end of surgery isoflurane and nitrous oxide were stopped and 100\% oxygen was given. As the patient regained consciousness, proseal LMA was removed. Patient was unable to close her mouth and had pain bilaterally in joint region. Manual reduction was attempted but was unsuccessful due to muscle tone of TMJ and pain during maneuver. Sevoflurane and nitrous oxide were started with sevoflurane at $1 \%$ and nitrous oxide and oxygen at $66 \%$ and $33 \%$ respectively. Patient was breathing spontaneously. Once patient was deeply sedated, downward and backward force was applied on mandible with both hands and complete reduction was achieved. A relocation clunck was felt bilaterally. Sevoflurane and nitrous oxide were stopped and patient was allowed to breathe $100 \%$ oxygen. As the patient achieved consciousness she was able to close her mouth comfortably without pain. ENT referral was done and she was evaluated for TMJ dysfunction. 


\section{Discussion}

TMJ dislocation is a known though rare complication during laryngoscopy for endotracheal intubation, LMA insertion and jaw thrust during mask ventilation. It is the dislodgement of mandibular condyle from its normal position in the squamotemporal portion of the cranial base. ${ }^{4,5}$ It can occur spontaneously during wide mouth opening. Wide mouth opening predisposes to bilateral anterior TMJ dislocation when condylar heads are forced to a position away from their resting position with in glenoid fossa. ${ }^{6}$ This may occur under general anaesthesia when muscles are relaxed. Prasad et $\mathrm{al}^{7}$ reported a case of TMJ dislocation during LMA insertion under general anaesthesia in a 65 year old male patient.TMJ dislocation occurred with wide opening of jaw to place oropharyngeal airway in mechanically ventilated patients undergoing laprotomy. ${ }^{8}$ Jaw thrust to lift the pharyngeal structures anteriorly facilitates passage of LMA into hypopharynx. ${ }^{9}$ Sia SL et a ${ }^{10}$ reported two cases of TMJ dislocation after LMA insertion under general anaesthesia in a 64 year male and 60 year female with no history of jaw dysfunction. Jaw thrust maneuver has been used to clear the airway and is recommended for LMA insertion ${ }^{11}$ and to assess the adequacy of depth of anaesthesia for LMA insertion. ${ }^{12}$

In present case jaw thrust maneuver was used in addition to standard technique that lead to anterior dislocation of TMJ. Dislocation was not recognized during LMA insertion and patient was ventilated comfortably without any complication.

\section{Conclusion}

TMJ dislocation is a rare side effect of airway manipulation, particularly in a sedated or anesthetized patient. Jaw thrust during LMA insertion can lead to TMJ dislocation even in normally functioning joint. Detailed history and thorough examination should be done to rule out any TMJ abnormality and possibility of dislocation during anaesthesia so that firm but gentle airway manipulation can be done in order to prevent dislocation. Extreme care should be taken during airway manipulation in patients with previous history of TMJ dislocation. TMJ should be routinely checked after LMA insertion to rule out TMJ disfunction.

\section{References}

1. Awsare AN, Prakash N. Temporomandibular dislocation: should every doctor be trained in resetting the jaw? $\mathrm{Br} \mathrm{J}$ Oral Maxillofac Surg. 2006;44:339.

2. Agro F, Salvinelli F. Temporomandibular joint assessment in anaesthetic practice. $\mathrm{Br} J$ Anaesth. 2003;7:707-708.

3. Insoo Han, Tae Kwane Kim. Dislocation of the temporomandibular joint following general anesthesia. Case report Korean J Anesthesiol. 2014;67:113-114.

4. Roze des Ordons A, Townsend DR. Trachlight management of succinycholine-induced subluxation of the temporo-mandibular joint: a case report and review of the literature. Can J Anaesth. 2008;55:616-621.

5. Akinbami BO. Evaluation of the mechanism and principles of management of temporomandibular joint dislocation. Systematic review of literature and a proposed new classification of temporomandibular joint dislocation. Head Face Med. 2011;7:10.

6. Luyk NH, Larsen PE. The diagnosis and treatment of the dislocated mandible. American Journal of Emergency Medicine. 1989;7:329-35.

7. Prasad G, Agarwal S. Temporomandibular joint dislocation during LMA insertion. Indian J Anaesth. 2004;48:151-152.

8. Sosis M, Lazar S. Jaw dislocation during general anaesthesia. Canadian Journal of Anaesthesia. 1987;34:407-8.

9. Aoyama K, Takenaka I. The triple airway manoeuvre for insertion of the laryngeal mask airway in paralyzed patients. Canadian Journal of Anaesthesia. 1995;42:1010-6.

10. Sia SL, Chang YL. Temporomandibular joint dislocation after laryngeal mask airway insertion. Acta Anaesthesiol Taiwan. 2008;46:82-85.

11. Fukotome T. Correct positioning of the epiglottis for application of the Brain laryngeal mask airway. Anaesthesia. 1995;50:818-19.

12. Drage MP, Nunez Z, Vaughan RS. Jaw thrusting as a clinical test to assess the adequate depth of anaesthesia for insertion of LMA. Anaesthesia. 1996;5:1167-70.

How to cite this article: Farooq Z, Gupta N, Nabi F. Intra operative temporomandibular joint dislocation during use of proseal LMA. Indian J Clin Anaesth. 2018;5(3):457-458. 